\title{
The Effect of CTV Shrinkage Margins in Treatment Planning Systems to the Breast Surface Doses
}

\author{
(1) Murat OKUTAN, ${ }^{1}$ (I) Nazan DEDE ${ }^{2}$ \\ 'Department of Medical Physics, İstanbul University Oncology Institute, İstanbul-Turkey \\ ${ }^{2}$ Department of Medical Physics, Dr. Lütfi Kırdar Kartal Training and Research Hospital, İstanbul-Turkey
}

\begin{abstract}
OBJECTIVE
This study aims to evaluate the skin dose variation on a breast phantom for patient with mastectomy treated with bolus intensity-modulated radiotherapy (IMRT) when the clinic target volume (CTV) shrinkage margin is $3 \mathrm{~mm}$ and $5 \mathrm{~mm}$.
\end{abstract}

\begin{abstract}
METHODS
Alderson Rando phantom computed tomography (CT) scan was performed for two situations: $1-\mathrm{cm}$ bolus and no-bolus. After the CTV organ at risk (OAR) volumes were created in the treatment planning system (TPS) using phantom image, no-bolus, 50\%-bolus, and 100\%-bolus IMRT plans were studied. The treatment plans for these three situations were made separately for 3-mm and 5-mm CTV shrinkage into the breast surface. The energy photon beams of $6 \mathrm{MV}$ were used for the treatment plans. The treatment plans were made using the IMRT technique to give a dose of $50 \mathrm{~Gy}$ in 25 fractions to CTV. Measurements were made with thin thermoluminescent dosimetry (TLD) chips. The TLD average readings and TPS readings at the same point were compared.
\end{abstract}

\section{RESULTS}

When the averages of the measurement data for 3-mm CTV shrinkage into the tissue are compared with the values obtained from the treatment planning system, the difference in surface doses for no-bolus, $50 \%$-bolus, and $100 \%$-bolus plans was determined as $20.3 \%, 18 \%$, and $12.6 \%$, respectively. For 5 -mm CTV shrinkage into the tissue, the difference in surface doses for no-bolus, $50 \%$-bolus, and $100 \%$-bolus plans was determined as $5.4 \%, 2.6 \%$, and $2.9 \%$, respectively.

\section{CONCLUSION}

We recommend that 5 - $\mathrm{mm}$ shrinkage with $50 \%$ bolus ( $1-\mathrm{cm}$ thickness) should be used for the better TPS surface dose calculation because the accuracy of TPS calculations increases with the decrease in differences between the TPS and TLD validation readings.

Keywords: Bolus; CTV shrinkage; IMRT; surface dose; TPS accuracy.

Copyright $\odot$ 2018, Turkish Society for Radiation Oncology

\section{Introduction}

For patients with mastectomy, postoperative radiotherapy is the preferred treatment modality to improve local control and survival.[1,2] Postmastectomy radiotherapy (PMRT) is usually performed with $3 \mathrm{D}$ confor- mal radiotherapy (3D-CRT) or intensity-modulated radiotherapy (IMRT) techniques using high-energy photons of 6 MV. $[1,3]$ In the PMRT, the chest wall and, if necessary, the lymph nodes form the target volume. Generally, the boost treatment is not applied to the PMRT.[1] A total dose of 45-50 Gy is given to the chest 
wall as a dose of 1.8-2 Gy per day-fraction.[4] When considering the possibility of cancer cells remaining in the skin, the skin tissue should be also included to the target volume in the breast-conserving surgery radiotherapy or PMRT applications.[1]

High-energy photons have skin-sparing-effect properties because they transmit their maximum energy to more depth than low-energy photons do. In this case, to treat the superficial lesions, a bolus is required to increase the maximum dose toward the surface.[5,6] Bolus is a tissue equivalent material that helps to smooth out the surface of the skin by filling in various tissue deficiencies in patients.[2,5] Although the bolus is used to increase the surface dose, the treatment-planning computers cannot correctly calculate the surface doses. Chung $\mathrm{H}$. et al. observed a reduction in treatment planning surface dose from $18.8 \%$ to $7.4 \%$ compared to that in the film surface dose. [7] And, the amount of CTV shrinkage into the tissue is controversial when tumor volume definition is performed in the treatment planning systems. [8,9]

In this study, we evaluated the skin dose variation on a breast phantom using the bolus IMRT technique when the amount of CTV shrinkage into the tissue is 3 $\mathrm{mm}$ and $5 \mathrm{~mm}$.

\section{Materials and Methods}

Target Volume Definitions and Treatment Planning For treatment planning of the phantom, Alderson Rando phantom (Supertech, USA) was placed on the computed tomography (CT) table at head-gantry and supine position. In phantom treatment plan applications, the projections of the lasers were marked on phantom surface, and the lead markers were placed in order on the phantom surface to make it easier to adjust the isocenter. Alderson Rando phantom CT scan was scanned for $1-\mathrm{cm}$ real bolus and no-bolus (virtual bolus) with 4-mm phantom slice thickness.

In the first part of the measurements, to determine the effect of $100 \%$ real bolus and $100 \%$ virtual bolus on the surface dose, bolus in all treatments plans were created as virtual and as real in treatment planning systems. Doses of 50 Gy were defined to CTV in 25 fractions. Treatment planning system was Eclipse (8.9, Varian Medical Systems, Palo Alto, CA, USA).

In the second part of the measurements, to determine the shrinkage effects on the surface dose for virtual bolus, CTV was separately created two times for 3- $\mathrm{mm}$ and 5-mm shrinkage. Three different plans were performed with no-bolus, $50 \%$ bolus, and $100 \%$ bolus. Doses of 50 Gy were defined to CTV in 25 fractions.
The energy photons of $6 \mathrm{MV}$ have a dmax of 1.5 $\mathrm{cm}$. In this study, the bolus thickness of $1 \mathrm{~cm}$ has been preferred because $1.5 \mathrm{~cm}$ thick bolus was not flexible enough in the clinical applications. The IMRT treatment fields were placed at $30^{\circ}$ intervals. Total seven fields were used, and the outermost and innermost fields were tangential.

\section{Dose Measurements:}

For the dose measurements, FIMEL (French) brand GR200 (3.2 mm diameter, $0.3 \mathrm{~mm}$ thick) TLDs placed on the Alderson Rando phantom surface were used. According to the manufacturer, the dose range of the dosimeters was between $0.5 \mu \mathrm{Gy}$ and $12 \mathrm{~Gy}$. The TLDs were read with a Fimel LTM manual TLD reader. They were calibrated using a 6-MV beam from a Varian Trilogy linear accelerator (Varian Medical Systems, Palo Alto, CA, USA) and RW3 solid water phantom (PTW, Freiburg, Germany). The TLDs are grouped so that the difference in reading values will be a maximum of $1 \%$.

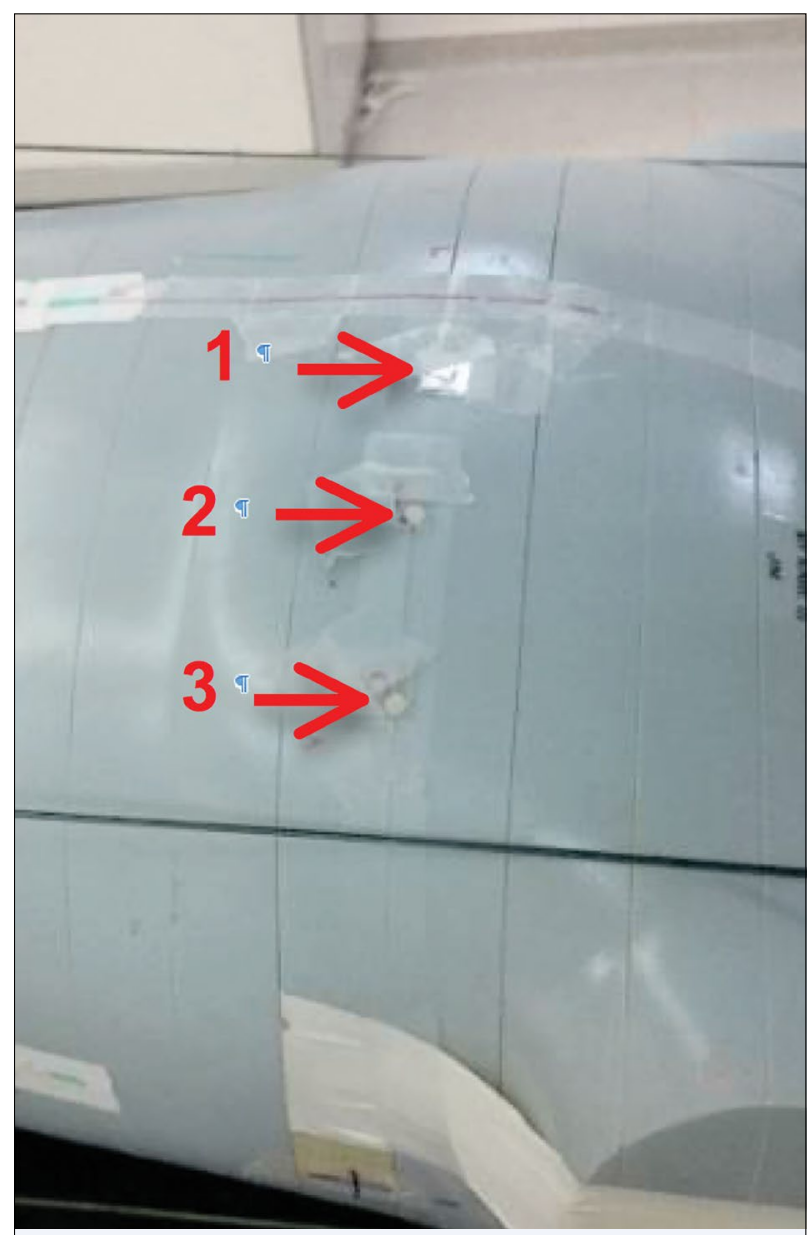

Fig. 1. TLD placement on Rando phantom. 


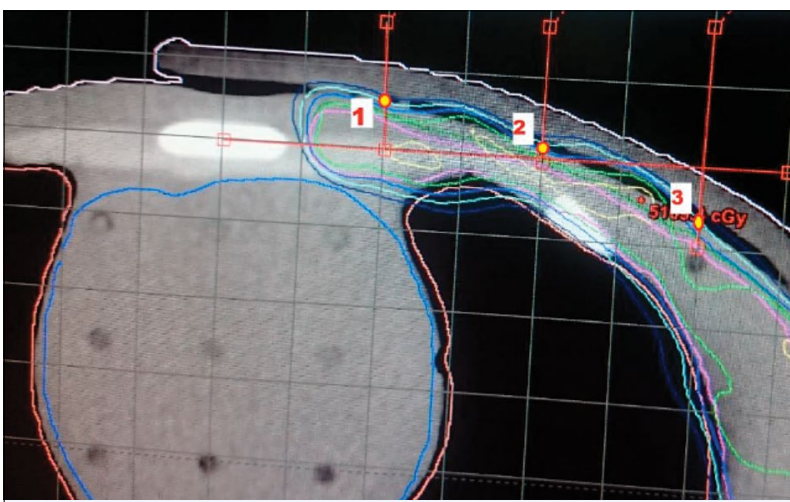

Fig. 2. Positions of TLDs in TPS.

During the irradiation, phantom was placed on the LINAC treatment table while paying attention to the control lines marked on the CT. With the help of table scrolling data from TPS, phantom was positioned to the treatment condition. The CT axial slices were used to determine the TLD positions on the phantom surface. The TLD chips were placed on these dotted places (Fig. 1). During the irradiations, a real bolus material, which was created in the TPS planning as virtual $(1-\mathrm{cm}$ thickness), was placed on the phantom. Each plan was irradiated three times, and the average readings were used for comparison. The mean TLD readings were compared with dose values at the same point on the treatment planning computer (Fig. 2).

\section{Results}

There are two different ways of making a bolus during the treatment planning phase: real bolus and virtual bolus. For treatment planning with the real bolus, a real bolus is placed on the chest wall during the patient's CT scan. On the other hand, for treatment planning with a virtual bolus, a virtual bolus is drawn on the skin in
TPS. In this study, the effect of both conditions on TPS skin dose was investigated with a margin of 3-mm and 5-mm CTV shrinkage margins.

In the first part of the study, only $100 \%$ virtual and $100 \%$ real bolus treatment situations were examined. Table 1 shows the effect of $100 \%$ real bolus and $100 \%$ virtual bolus on the surface dose between the TPS values and TLD validation measurements. As shown in Table 1, there is no significant difference between the real and virtual situation. It was determined that there is $1 \%$ difference between the TPS values and TLD validation measurements for 3-mm shrinkage, and there is $0.5 \%$ difference between the TPS values and TLD validation measurements for 5-mm shrinkage.

In the second part of the study, the no-bolus $(0 \%$ virtual bolus and $0 \%$ real bolus) situation, $50 \%$ virtual bolus, and $100 \%$ virtual bolus cases were examined. The values obtained from the measurements are given in Table 2. Table 2 shows the mean values and differences between the TLD validation measurements and TPS calculations on CTV with 3-mm shrinkage. The differences between the TPS and TLD validation readings are $20.3 \%, 18.0 \%$, and $12.6 \%$ for no-bolus, $50 \%$ bolus, and $100 \%$ bolus, respectively.

As similar, for CTV with 5-mm shrinkage, Table 2 shows the mean values and differences between the same points in the TLD and TPS. The differences between the TPS and TLD validation measurements are $5.4 \%, 2.6 \%$, and $2.9 \%$ for no-bolus, $50 \%$ bolus, and $100 \%$ bolus, respectively. The minimum difference between the TPS and TLD average readings for 5-mm shrinkage was $2.6 \%$ in $50 \%$ bolus plan.

\section{Discussion}

Yokoyama S. et al. performed several phantom measurements with $6 \mathrm{MV}$-energized photons using con-

Table 1 The TPS surface dose comparisons of 100\% real bolus and 100\% virtual bolus plans using the TLD validation

\section{Average Value (cGy) \\ $3 \mathrm{~mm}$ CTV shrinkage margins}

\begin{tabular}{lc}
\hline TPS 100\% Real Bolus & 358.35 \\
Validation with TLD & 310.46 \\
Difference (\%) & 15.4 \\
& Aver \\
& $\mathbf{5 ~ m m ~ C T V}$ \\
TPS 100\% Real Bolus & 344.56 \\
Validation with TLD & 314.24 \\
Difference (\%) & 9.6
\end{tabular}

Average Value (cGy)
366.23

320.17

14.4
TPS 100\% Virtual Bolus
Validation with TLD
Difference (\%)
371.29

340,48

9.1 
Table 2 TPS surface dose comparisons for no-bolus, 50\% virtual bolus, and $100 \%$ virtual bolus plans using the TLD validation

$3 \mathrm{~mm}$ shrinkage margins

\begin{tabular}{|c|c|c|c|c|c|}
\hline TPS no-bolus & 244.87 & TPS $50 \%$ Virtual Bolus & 305.27 & TPS $100 \%$ Virtual Bolus & 366.23 \\
\hline Validation with TLD & 195.05 & Validation with TLD & 250.45 & Validation with TLD & 320.17 \\
\hline Difference(\%) & $20.3 \%$ & Difference(\%) & $18 \%$ & Difference(\%) & 12.6 \\
\hline \multicolumn{6}{|c|}{$5 \mathrm{~mm}$ shrinkage margins } \\
\hline TPS no-bolus & 194.48 & TPS 50\% Virtual bolus & 278.48 & TPS $100 \%$ Virtual bolus & 360.48 \\
\hline Validation with TLD & 205.53 & Validation with TLD & 271.16 & Validation with TLD & 371.29 \\
\hline Difference(\%) & 5.4 & Difference(\%) & 2.6 & Difference(\%) & 2.9 \\
\hline
\end{tabular}

formal open field and IMRT fields.[10] They observed that the surface dose in the IMRT fields was $10 \%$ lower than in the conformal open fields. On the other hand, in dosimetric measurements for surface doses of 6-10 MV photons, Laurence E. Court et al. found a reduction of up to $20 \%$ in the Eclipse treatment planning doses compared to the values measured for the IMRT plans.[11] These studies show that the dose received by the skin in the IMRT plans is lower than the dose prescribed for the target. Therefore, a convenient option is to use a bolus material to achieve an enough dose in the skin.[8]

$100 \%$ bolus treatment is not preferred because the skin recieves too much radiation. Because the skin receives too much radiation, $100 \%$ bolus treatment is not preferred. Therefore, a part of the treatment is irradiated using a bolus. In an international study conducted by T.T.T. Vu et al. in 2007, preferences of world clinics regarding the use of bolus material in the PMRT treatments have been shown.[12] A total of $87.5 \%$ of the PMRT treatments are performed with high-energy photons, $9 \%$ with electrons, $1 \%$ with Co-60 source, and the remaining $2.5 \%$ with photon-electron combination. A total of $68 \%$ of the clinics stated that bolus material was used in all PMRT treatments, $6 \%$ of clinics stated that bolus material was not used, and $26 \%$ of clinics stated that they were behaving according to the situation. A total of $33 \%$ of the clinics who prefer a bolus material use a bolus in each fraction $(100 \%$ bolus), and $48 \%$ of clinics prefer one bolus in two fractions (50\% bolus).[12] In our clinic, we also perform a PMRT radiotherapy with $50 \%$ bolus.

An-Cheng S. et al.[9] reported that the accuracy of the surface dose depends on the TPS calculation in the head and neck IMRT plans. They demonstrated that the difference between the measurement and the calculation is more than $10 \%$ in case of $5-\mathrm{mm}$ CTV shrinkage margins. As the depth increases, the accuracy of the calculation improves. For example, the value is between $2.5 \%$ and $5.5 \%$ for $7-\mathrm{mm}$ CTV shrinkage margin. They also emphasized that when the tumor invaded to the superficial region as breast cancer, the bolus was the best way to deliver a sufficient dose.

\section{Conclusion}

We recommend that $5-\mathrm{mm}$ shrinkage with $50 \%$ bolus (1-cm thickness) should be used for the better TPS surface dose calculation because the accuracy of TPS calculations increases with the decrease in differences between the TPS and TLD validation readings. Because there is no significant difference between the TPS calculation and the TLD validation measurements for real and virtual bolus, virtual bolus can be used instead of real bolus.

Peer-review: Externally peer-reviewed.

Conflict of Interest: The authors declare that there is no conflict of interest.

\section{Financial Support: None.}

Authorship contributions: Concept - M.O.; Design M.O., N.D.; Supervision - M.O.; Materials - M.O., N.D.; Data collection \&/or processing - N.D., M.O.; Analysis and/ or interpretation - M.O., N.D.; Literature search - N.D.; Writing - M.O.; Critical review - M.O.

\section{References}

1. Barrett A, Dobbs J, Morris S, Roques T. Practical Radiotherapy Planning. 4th ed. London: Hodder Arnold; 2009.

2. Halperin CE, Perez AC, Brady LW. Perez and Brady's principles and practice of radiation oncology. 5th ed. Philadelphia: Lippincott Williams \& Wilkins; 2008.

3. Fischbach M, Hälg RA, Hartmann M, Besserer J, Gruber G, Schneider U. Measurement of skin and target dose in post-mastectomy radiotherapy using 4 and 6 MV photon beams. Radiat Oncol 2013;8:270. 
4. Hansen EK, Roach M. Handbook of Evidence-Based Radiation Oncology. 2nd ed. New York: Springer; 2010.

5. Kahn YK, Villareal-Barajas E, Udowicz M, Sinha R, Muhammad W, Abbasi AN, et al. Clinical and dosimetric implications of air gaps between bolus and skin surface during radiation therapy. Journal of Cancer Therapy 2013;4:1251-5.

6. Kahn FM. The Physics of Radiation Therapy. 4th ed. Philadelphia: Lippincott Williams \& Wilkins; 2010.

7. Chung H, Jin H, Dempsey JF, Liu C, Palta J, Suh TS, et al. Evaluation of surface and build-up region dose for intensity-modulated radiation therapy in head and neck cancer. Med Phys 2005;32(8):2682-9.

8. Shiau AC, Chiu MC, Chen TH, Chiou JF, Shueng PW, Chen SW, et al. Surface and superficial dose dosimetric verification for postmastectomy radiotherapy. Med Dosim 2012;37(4):417-24.
9. Shiau AC, Lai PL, Liang JA, Shueng PW, Chen WL, Kuan WP. Dosimetric verification of surface and superficial doses for head and neck IMRT with different PTV shrinkage margins. Med Phys 2011;38(3):143543.

10. Yokoyama S, Roberson PL, Litzenberg DW, Moran JM, Fraass BA. Surface buildup dose dependence on photon field delivery technique for IMRT. J Appl Clin Med Phys 2004;5(2):71-81.

11. Court LE, Tishler R, Xiang H, Allen AM, Makrigiorgos M, Chin L. Experimental evaluation of the accuracy of skin dose calculation for a commercial treatment planning system. J Appl Clin Med Phys 2008;9(1):2792.

12. Vu TT, Pignol JP, Rakovitch E, Spayne J, Paszat L. Variability in radiation oncologists' opinion on the indication of a bolus in post-mastectomy radiotherapy: an international survey. Clin Oncol (R Coll Radiol) 2007;19(2):115-9. 